\title{
Transcriptional upregulation of both egl-1 BH3-only and ced-3 caspase is required for the death of the male-specific CEM neurons
}

\author{
R Nehme ${ }^{1}$, P Grote ${ }^{1,2}$, T Tomasi ${ }^{1,4}$, S Löser ${ }^{2,4}$, H Holzkamp $^{2}$, R Schnabel ${ }^{3}$ and B Conradt ${ }^{\star, 1}$
}

Most of the 131 cells that die during the development of a Caenorhabditis elegans hermaphrodite do so $\sim 30$ min after being generated. Furthermore, in these cells, the pro-caspase proCED-3 is inherited from progenitors and the transcriptional upregulation of the BH3-only gene egl-1 is thought to be sufficient for apoptosis induction. In contrast, the four CEM neurons, which die in hermaphrodites, but not males, die $\sim 150$ min after being generated. We found that in the CEMs, the transcriptional activation of both the egl-1 and ced-3 gene is necessary for apoptosis induction. In addition, we show that the Bar homeodomain transcription factor CEH-30 represses egl-1 and ced-3 transcription in the CEMs, thereby permitting their survival. Furthermore, we identified three genes, unc-86, Irs-1, and unc-132, which encode a POU homeodomain transcription factor, a leucyl-tRNA synthetase, and a novel protein with limited sequence similarity to the mammalian proto-oncoprotein and kinase PIM-1, respectively, that promote the expression of the ceh-30 gene in the CEMs. On the basis of these results, we propose that egl-1 and ced-3 transcription are coregulated in the CEMs to compensate for limiting proCED-3 levels, which most probably are a result of proCED-3 turn over. Similar coregulatory mechanisms for BH3-only proteins and pro-caspases may function in higher organisms to allow efficient apoptosis induction. Finally, we present evidence that the timing of the death of the CEMs is controlled by TRA-1 Gli, the terminal global regulator of somatic sexual fate in $C$. elegans.

Cell Death and Differentiation (2010) 17, 1266-1276; doi:10.1038/cdd.2010.3; published online 12 February 2010

Owing to its invariant pattern of apoptotic cell death during development, Caenorhabditis elegans has become an important model for apoptosis studies. The central cell death machinery in $C$. elegans is composed of the anti-apoptotic BCL-2-like protein CED-9, the APAF-1-like caspase activator CED-4 and the pro-caspase proCED-3. ${ }^{1,2}$ At least during midembryogenesis when the majority of cell deaths occur, most if not all cells in developing embryos contain CED-4 as well as proCED-3, which once matured is sufficient for apoptosis induction. $^{3-5}$ However, these cells also contain the CED-9 protein, which through its direct interaction with CED-4, blocks the ability of CED-4 to mediate proCED-3 maturation. ${ }^{3,4}$ As a result, apoptosis induction is prevented.

During the development of a $C$. elegans hermaphrodite, exactly 131 cells are programmed to die. ${ }^{6}$ The majority of these cells die $\sim 30$ min after being generated. Furthermore, in these cells, the transcriptional activation of the $\mathrm{BH} 3-$ only gene egl-1 is thought to be sufficient for the activation of the central cell death machinery and, hence, apoptosis induction. ${ }^{7}$ Specifically, the BH3-only protein EGL-1 can bind to CED-9 thereby causing CED-4 release from CED-9 and CED4-dependent proCED-3 maturation. Interestingly, recent data suggest that the ced-3 gene is not transcriptionally active in most of the 131 cells that are programmed to die during development. ${ }^{4}$ This observation suggests that proCED-3 protein present in these cells is derived from their progenitors. It also suggests that once activated, the amount of proCED-3 protein inherited from progenitors is, in general, sufficient for apoptosis induction.

The four male-specific cephalic companion neurons (CEMs) are generated $\sim 320$ min after the first cell division. In males, the CEMs survive and differentiate into ciliated sensory neurons implicated in mating behavior.,8,9 In hermaphrodites, however, the CEMs undergo apoptosis $\sim 150$ min after being generated $(\sim 470 \mathrm{~min}$ after the first cell division). As is the case for most of the cells that are programmed to die during development, the death of the CEMs is dependent on the genes egl-1, ced-4, and ced-3. ${ }^{10,11}$ The death of the CEMs is also dependent on the gene tra-1, which encodes the terminal, global regulator of somatic sexual fate, and which acts to promote female development. ${ }^{12}$ Specifically, the TRA-1 protein, a GLI-like transcription factor, causes the CEMs in hermaphrodites to undergo apoptosis by directly repressing the transcription of the gene ceh-30, which encodes a Bar homeodomain transcription factor that acts to block the death of the CEMs in males. ${ }^{13,14}$

How the CEH-30 protein acts on the central cell death machinery to block the death of the CEMs has so far been

\footnotetext{
${ }^{1}$ Department of Genetics, Dartmouth Medical School, Norris Cotton Cancer Center, Hanover, NH, USA; ${ }^{2}$ Max-Planck-Institute of Neurobiology, Am Klopferspitz, Martinsried, Germany and ${ }^{3}$ Institut für Genetik, Technische Universität Braunschweig Carolo Wilhelmina, Braunschweig, Germany

${ }^{*}$ Corresponding author: B Conradt, Department of Genetics, Dartmouth Medical School, 7400 Remsen Building, Hanover, NH 3755 , USA. Tel: 603 650 1210;

Fax: 603650 1188; E-mail: barbara.conradt@dartmouth.edu

${ }^{4}$ These authors contributed equally to this work.

Keywords: BH3-only gene; caspase gene; apoptosis induction; developmental control; $C$. elegans

Abbreviations: CED, cell-death abnormal; EGL, egg-laying abnormal; CEM, cephalic companion neurons; UNC, uncoordinated

Received 12.2.09; revised 02.11.09; accepted 30.11.09; Edited by M Hengartner; published online 12.2.10
} 
unclear. We now present evidence that $\mathrm{CEH}-30$ blocks the death of the CEMs by repressing the transcription of both the egl-1 and ced-3 gene. Furthermore, we identified three genes, unc-86, Irs-1, and unc-132, which encode a POU homeodomain transcription factor, a leucyl-tRNA synthetase, and a novel protein with limited sequence similarity to the mammalian proto-oncoprotein and kinase PIM-1, respectively, that promote ceh-30 expression in the CEMs. Our data show that in contrast to most cells that are programmed to die during development, in the CEMs, the transcriptional upregulation of both the BH3-only gene egl-1 and the caspase gene ced-3 is required for apoptosis induction. We propose that coupling the transcriptional upregulation of a key activator of apoptosis induction (egl-1) and a component of the central, enzymatic cell death machinery (ced-3) ensures efficient apoptosis induction in the CEMs.

\section{Results}

bc151, bc155, and bc159 cause differentiated CEMs to be absent from masculinized hermaphrodites or males. An altered-function mutation of the $C$. elegans gene sel-10, n1077, which encodes an F-box protein that promotes female development, causes weak masculinization of the hermaphrodite soma. ${ }^{15-17}$ For example, in wild-type hermaphrodites, the four CEMs undergo apoptosis. ${ }^{6,8}$ In contrast, in sel-10(n1077) hermaphrodites, the CEMs survive like in wild-type males. To identify genes required for the survival of the CEMs, we sought mutations that cause the CEMs to be absent from sel-10(n1077) hermaphrodites based on the expression of $p k d-2$, a marker for differentiated CEMs (see Materials and Methods). We identified three recessive mutations, bc151, bc155, and bc159, that cause the majority of CEMs to be absent from masculinized hermaphrodites as well as males (Table 1a; data not shown). The CEMs are also absent in bc151, bc155, or bc159 masculinized hermaphrodites based on their characteristic morphology in L4 larvae (Table 1b) and in bc151, bc155, or bc159 males based on lov-1 expression, another marker for differentiated CEMs (Supplementary Table S1). In addition, all three mutations cause a reduced brood size and an uncoordinated or 'Unc' phenotype (Supplementary Table S2; data not shown). On the basis of these observations, we conclude that bc151, bc155, and bc159 cause differentiated CEMs to be absent in masculinized hermaphrodites and males.

bc151 is a loss-of-function mutation of unc-86 POU. unc-86, which encodes a POU transcription factor, has previously been shown to be required for the presence of differentiated CEMs in males. ${ }^{13,18,19}$ We found that bc151 animals carry two mutations in the unc- 86 gene: first, a missense mutation leading to an aspartic acid (GAU)-toasparagine (AAU) substitution at position 203 of the protein sequence of the UNC-86.b protein (generated by transcript C30A5.7b); second, a nonsense mutation, changing codon 220 of the C30A5.7b coding sequence (CAA) to a STOP codon (UAA) (Figure 1a). Both unc-86 transcripts (C30A5.7a, $C 30 A 5.7 b)$ are predicted to be affected by the two mutations. As loss-of-function mutations of unc- 86 have previously been shown to cause differentiated CEMs to be absent in males, we conclude that $b c 151$ is a new loss-of-function mutation of the unc-86 gene.

Table 1 The CEMs are absent in masculinized hermaphrodites or males homozygous for unc-86(bc151), Irs-1(bc155) or unc-132(bc159).

\begin{tabular}{|c|c|c|c|c|}
\hline \multirow[b]{3}{*}{ Genotype } & \multicolumn{4}{|c|}{$\%$ GFP $^{+}$CEMs (n) } \\
\hline & \multicolumn{2}{|c|}{$+/+$} & \multicolumn{2}{|c|}{ sel-10(n1077) } \\
\hline & $\mathbf{X X}$ & $x O^{a}$ & $+/+X X$ & ced-3(n717) XX \\
\hline \multicolumn{5}{|c|}{ (a) Presence of differentiated CEMs in adults } \\
\hline$+/+$ & 0 (Many) & 100 (Many) & 100 (Many) & 100 (Many) \\
\hline egl-1(n1084 n3082) & $68(356)$ & ND & ND & ND \\
\hline ced-3(n717) & $78(232)$ & ND & ND & ND \\
\hline$u n c-86(b c 151)^{b}$ & ND & $25(80)$ & $12(178)$ & $8(92)$ \\
\hline Irs-1(bc155) $)^{\mathrm{b}}$ & ND & $18(76)$ & $10(224)$ & $7(80)$ \\
\hline unc-132(bc159) & ND & $0(68)$ & $0(232)$ & $0(96)$ \\
\hline
\end{tabular}

$\%$ CEMs (n)

\begin{tabular}{|c|c|c|c|}
\hline Genotype & $+/+X X$ & sel-10(n1077) XX & ced-3(n717); sel-10(n1077) XX \\
\hline \multicolumn{4}{|c|}{ (b) Presence of CEMs in L4 larvae } \\
\hline$+/+$ & $0(36)$ & $100(40)$ & $100(36)$ \\
\hline egl-1(n1084 n3082) & $96(80)$ & ND & ND \\
\hline ced-3(n717) & $98(128)$ & ND & ND \\
\hline$u n c-86(b c 151)^{\mathrm{b}}$ & ND & $6(32)$ & $4(24)$ \\
\hline Irs-1(bc155) $)^{b}$ & ND & $7(48)$ & $7(48)$ \\
\hline unc-132(bc159) & ND & $0(20)$ & $2(44)$ \\
\hline
\end{tabular}

The presence of differentiated CEMs was analyzed in adults using the $\mathrm{P}_{p k d-2}$ gfp reporter as described in Materials and Methods (part (a)). The presence of CEMs was scored at the L4 stage using DIC as described in Materials and Methods (part (b)). All strains were homozygous for the $\mathrm{P}_{p k d-2}$ gfp integration bcls9. ${ }^{\text {Indicates that }}$ the strains were homozygous for him-5(e1490) V. 'Indicates that the strains were homozygous for dpy-17(e164) III. 
a

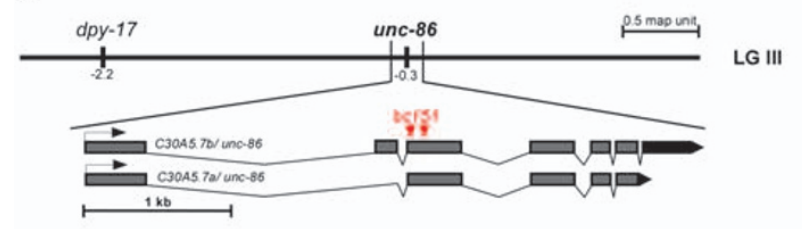

C

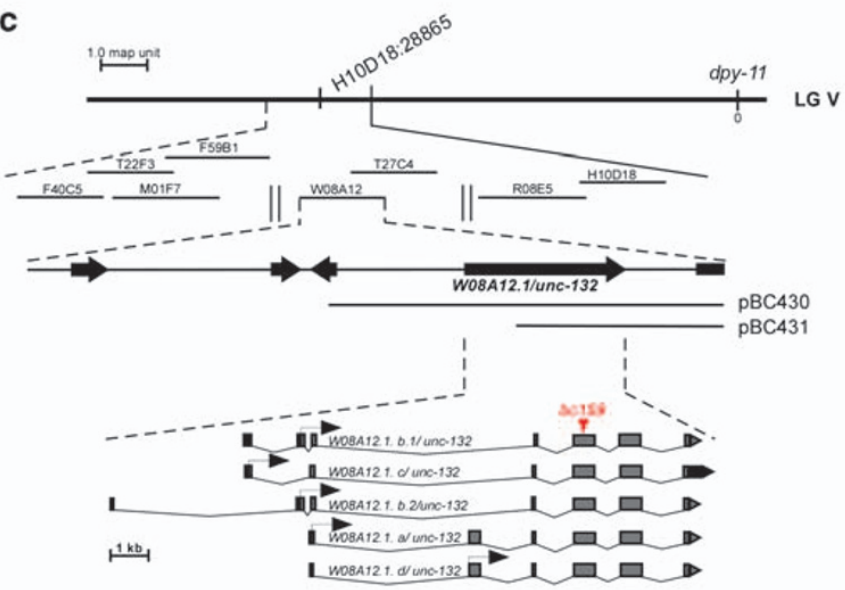

b

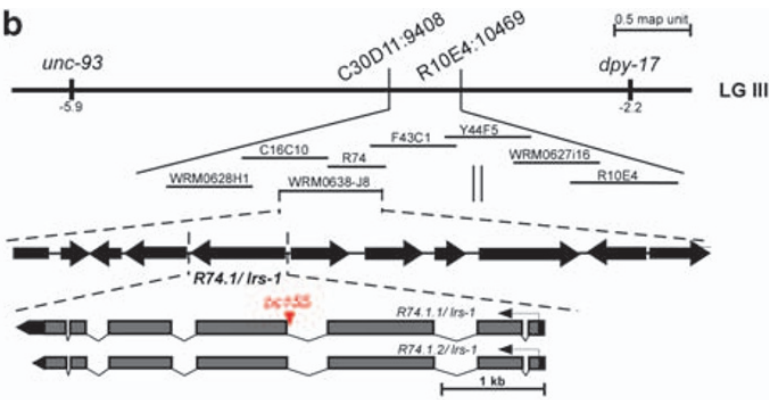

d

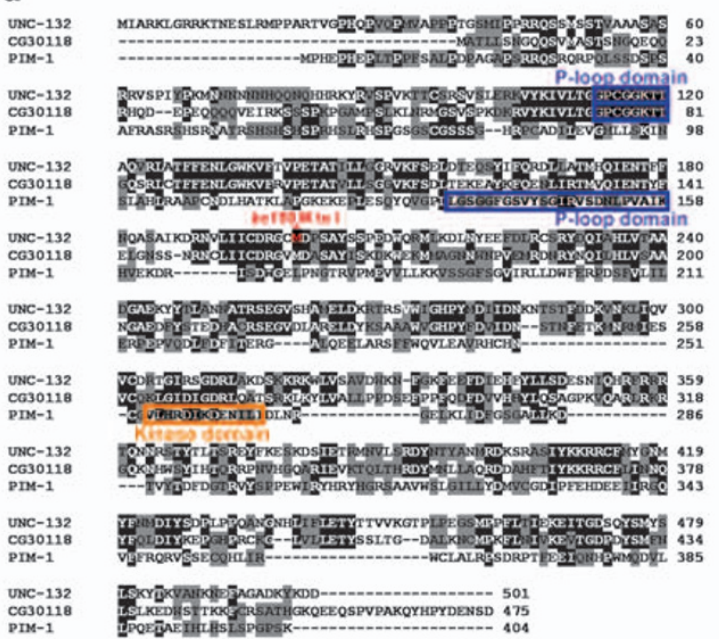

Figure 1 Cloning of unc-86(bc151), Irs-1(bc155), and unc-132(bc159). (a) The gene dpy-17 used for mapping bc151 is indicated on the genetic map (LG III). The two mutations identified in the C30A5.7/unc-86 gene in bc151 animals are indicated in red. (b) Genes and SNPs used for mapping bc155 on LG III are indicated on the genetic map. Cosmids and fosmids tested for bc155 rescue are shown below the genetic map. The bc155 mutation in R74.1//rs-1 is indicated in red. (c) dpy-11 and H10D18:28865 used for mapping bc159 on LG V are indicated on the genetic map. Cosmids tested for bc159 rescue are shown below. pBC430 and pBC431 represent rescuing subclones of cosmid W08A12. The bc159 mutation in W08A12.1/unc-132 is indicated in red. (d) Alignment of the protein sequences of UNC-132 (isoform a), D. melanogaster CG30118 and human PIM-1. The alignment was done using the EMBOSS algorithm. Identical amino acids have a black background. Amino acids with similar biochemical properties have a gray background. The blue boxes indicate the P-loop domains and the orange box the kinase domain. The amino acid that is changed as a result bc159 (M200I) is indicated with a red asterisk. Over its entire length, the UNC-132 protein is $38.2 \%$ identical and $53.5 \%$ similar to the CG30118 protein. The similarities between UNC-132 and CG30118 are mainly found in the central ( $57.5 \%$ identity, $76.4 \%$ similarity) and C-terminal (42.3\% identity, $59.6 \%$ similarity) regions of the two proteins. Over its entire length, the UNC-132 protein is $12.7 \%$ identical and $22.0 \%$ similar to the human kinase PIM-1. The similarity between the two proteins is mainly restricted to their $\mathrm{N}$-terminal (29.9\% identical, $46.8 \%$ similar) regions

bc155 is a loss-of-function mutation of the gene Irs-1 leucyl-tRNA synthetase. We found that bc155 is a mutation in the gene Irs-1, which encodes a leucyl-tRNA synthetase, a Class I tRNA synthetase (Figure 1b; data not shown). ${ }^{20}$ bc155 is a G-to-A transition that changes the $3^{\prime}$ splice acceptor site of intron 3 from $A G$ to $A A$, which results in reduced levels of fully spliced Irs-1 mRNA (Figure 1b; data not shown). Both Irs-1 transcripts (R74.1.1, R74.1.2) are predicted to be affected by the bc155 mutation. Reducing Irs-1 function by RNAi results in the absence of differentiated CEMs in masculinized hermaphrodites (Table 2a). On the basis of these findings, we conclude that $b c 155$ is a loss-offunction mutation of the gene Irs-1. Furthermore, we found that reducing the function by RNAi of genes encoding other Class I or Class II tRNA synthetases can result in the absence of at least $20 \%$ of differentiated CEMs in masculinized hermaphrodites (Table 2a).

bc159 is a loss-of-function mutation of the PIM-1-like gene unc-132. bc159 is a mutation in a previously uncharacterized gene, W08A12.1, which generates five transcripts. Transcript W08A12.1.a encodes a 501 aminoacid protein of unknown function with a $\mathrm{P}$-loop domain (Figure 1c). ${ }^{21,22}$ As bc159 also causes an Unc phenotype, we named W08A12.1 'unc-132. The bc159 mutation affects the second exon that is common to all five unc-132 transcripts. Specifically, bc159 is a missense mutation that leads to a methionine (AUG)-to-isoleucine (AUA) substitution at position 200 of the protein sequence of the UNC-132.a protein (generated by transcript W08A12.1.a). The reduction of unc-132 function by RNAi causes differentiated CEMs to be absent in a sel-10(n1077) background (Table 2b), confirming that $b c 159$ is a loss-of-function mutation of the unc-132 gene. unc-132 is orthologous to a gene of unknown function in Drosophila melanogaster, CG30118. ${ }^{21}$ Among mammalian proteins, UNC-132 is most similar to the human kinase PIM-1; however, the two proteins are not orthologous. ${ }^{22}$ As is the case for UNC-132 and CG30118, PIM-1 has a P-loop domain. However, in contrast to UNC-132 and CG30118, PIM-1 also contains a kinase domain. 
Table 2 The inactivation by RNAi of Irs-1 and other genes encoding tRNA synthetases as well as unc-132 by RNAi results in the absence of differentiated CEMs in masculinized hermaphrodites.

\begin{tabular}{|c|c|c|c|}
\hline Genotype & tRNA synthetase affected & $\% \mathrm{GFP}^{+}$CEMs (n) sel-10(n1077) XX & Other RNAi phenotypes \\
\hline \multicolumn{4}{|c|}{ (a) Inactivation by RNAi of Irs-1 and other genes encoding $t R N A$ synthetases } \\
\hline \multicolumn{4}{|c|}{ Class I tRNA synthetases } \\
\hline Irs-1(RNAi) & Leu & $62(60)$ & Larval arrest, lethality \\
\hline irs-2(RNAi) & lle & $63(248)$ & None detected \\
\hline irs-1(RNAi) & Ile & $83(12)$ & Larval arrest, lethality \\
\hline vrs-2(RNAi) & Val & $60(32)$ & Larval arrest, lethality \\
\hline vrs-1(RNAi) & Val & $90(124)$ & None detected \\
\hline$m r s-1(R N A i)$ & Met & $89(132)$ & None detected \\
\hline \multicolumn{4}{|c|}{ Class II tRNA synthetases } \\
\hline hrs-1(RNAi) & His & $71(24)$ & Larval arrest, lethality \\
\hline srs-1(RNAi) & Ser & $95(112)$ & None detected \\
\hline yrs-1(RNAi) & Tyr & $88(68)$ & None detected \\
\hline ers-1(RNAi) & Glu & $85(100)$ & None detected \\
\hline$w r s-2(R N A i)$ & Trp & $75(124)$ & None detected \\
\hline$d r s-1(R N A i)$ & Asp & $92(24)$ & Larval arrest, lethality \\
\hline prs-1(RNAi) & Pro & $97(116)$ & None detected \\
\hline ars-1(RNAi) & Ala & $88(124)$ & None detected \\
\hline $\operatorname{trs}-1(R N A i)$ & Thr & $74(72)$ & None detected \\
\hline$n r s-1(R N A i)$ & Asn & $86(28)$ & None detected \\
\hline frs-1(RNAi) & Phe & $100(36)$ & Larval arrest, lethality \\
\hline crs-1(RNAi) & Cys & $91(108)$ & Larval arrest, lethality \\
\hline$k r s-1(R N A i)$ & Lys & $100(32)$ & Larval arrest, lethality \\
\hline
\end{tabular}

Genotype

\% GFP ${ }^{+}$CEMs (n) sel-10(n1077)

(b) Inactivation of unc-132 by RNAi

$+/+$

99 (200)

unc-132(RNAi; W08A12.1b cDNA)

49 (216)

unc-132(RNAi ; W08A12.1c cDNA)

64 (172)

The presence of differentiated CEMs was analyzed in adults using the $\mathrm{P}_{p k d-2}$ gfp reporter as described in Materials and Methods. All strains were homozygous for the

$\mathrm{P}_{p k d-2}$ gfp integration bcls9. RNAi was performed by feeding as described in Materials and Methods.

Table 3 The fate of the AMso and URAs in unc-86(bc151), Irs-1(bc155) and unc-132(bc159)

\begin{tabular}{|c|c|c|c|}
\hline Genotype & $\% \mathrm{GFP}^{+}$AMso (n) $\mathrm{P}_{\text {unc-53 }}$ gfp & $\%$ GFP $^{+}$URAs (n) $P_{g l r-4} g r l-4:: g f p$ & $\%$ GFP $^{+}$URAs (n) $P_{c f i-1} g f p$ \\
\hline $\begin{array}{l}++ \\
\text { unc-86(bc151) } \\
\text { Irs-1(bc155) } \\
\text { unc-132(bc159) }\end{array}$ & $\begin{array}{l}100(120) \\
100(100) \\
100(140) \\
100(140)\end{array}$ & $\begin{array}{r}95(92) \\
8(40) \\
93(84) \\
77(88)\end{array}$ & $\begin{array}{c}91(124) \\
0(100) \\
89(60) \\
93(60)\end{array}$ \\
\hline
\end{tabular}

The presence of the AMso and URAs was determined in hermaphrodites using $\mathrm{P}_{\text {unc-53 }}$ gfp (bgEx21) (AMso) or $\mathrm{P}_{\text {glr-4 }}$ grl-4::gfp (akEx32) and $\mathrm{P}_{\text {cfi- }}$ gfp (nsEx37) (URAs) as described in Materials and Methods. Strains carrying the array akEx32 were homozygous for lin-15(n765ts) X. Strains carrying the array nsEx37 were homozygous for him-5(e1490) V and lin-15(n765ts) X. Indicates that the strains were homozygous for dpy-17(e164) III.

The loss of unc-86, Irs-1, or unc-132 function causes a defect in CEM specification. Next, we determined the fate of the CEMs in masculinized hermaphrodites lacking unc-86, Irs-1, or unc-132 function. Briefly, a mutation that blocks apoptotic cell death in general (ced-3(n717)) failed to suppress the CEM phenotype observed in these mutant backgrounds, which indicates that the CEMs do not inappropriately undergo apoptosis (Tables $1 \mathrm{a}$ and $\mathrm{b}$ ). In addition, we found that the loss of Irs-1 or unc-132 function does not affect the presence of differentiated dorsal URAs or amphid socket cells, the sisters of the dorsal CEMs and ventral CEMs, respectively, ${ }^{6}$ which indicates that the CEMs are generated (Table 3). Finally, as shown below, we can detect CEMs in unc-86 mutant embryos using a transcriptional egl-1 reporter.
Therefore, we conclude that the CEMs are also generated in animals lacking unc-86 function. On the basis of these observations, we propose that masculinized hermaphrodites lacking unc-86, Irs-1, or unc-132 function lack differentiated CEMs as a result of a defect in the specification (rather than generation or survival) of the CEMs.

unc-86, Irs-1, and unc-132 are required for ceh-30 expression in the CEMs in masculinized hermaphrodites. The Bar homeodomain transcription factor $\mathrm{CEH}-30$, whose expression is under the direct control of the terminal, global regulator of somatic sexual fate, TRA-1, acts to block the death of the CEMs. ${ }^{13,14}$ Specifically, the loss of ceh-30 function causes the CEMs to inappropriately die in 


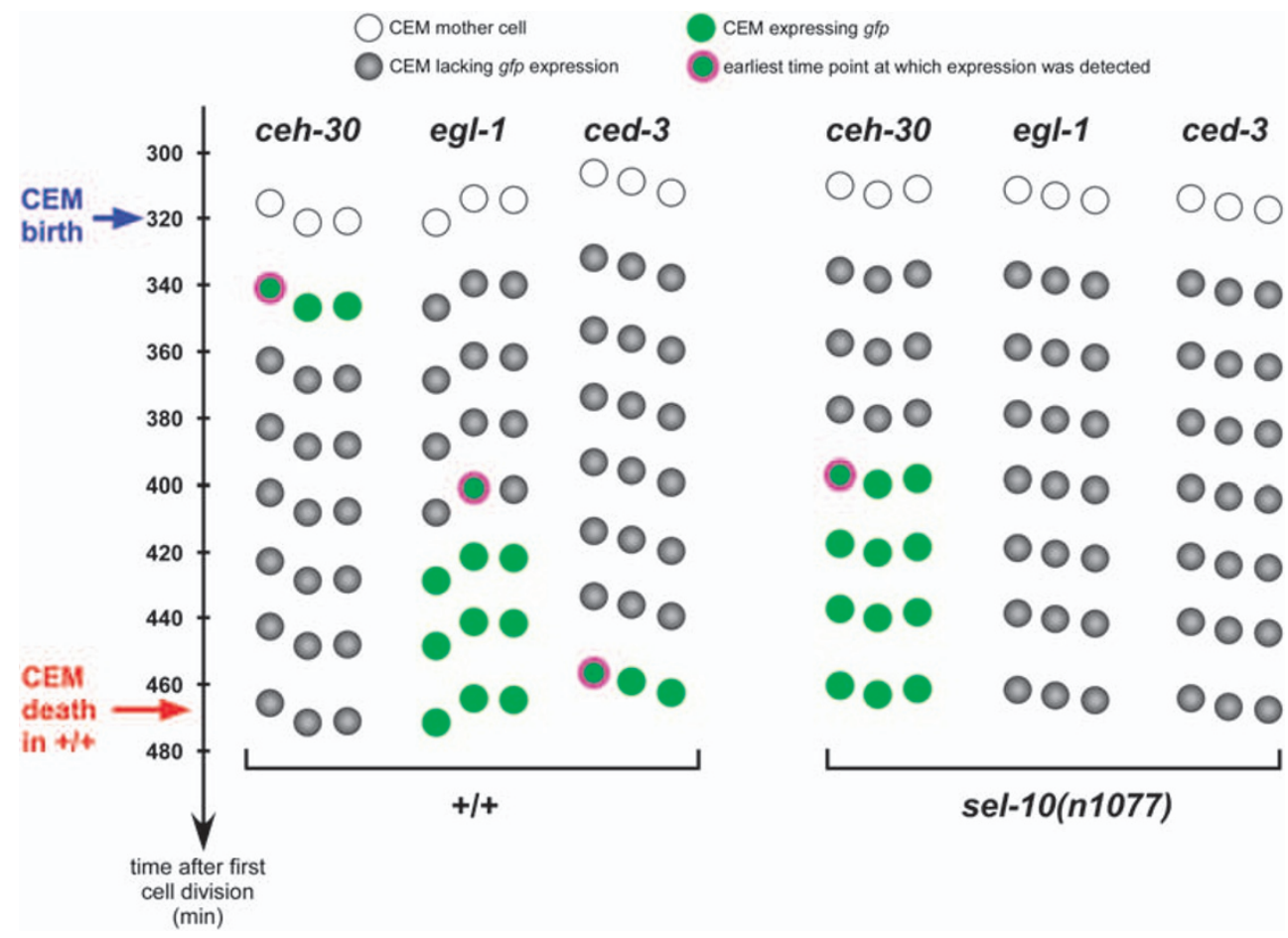

Figure 2 ceh-30, egl-1, and ced-3 expression in embryonic CEMs by 4D microscopy and lineaging analysis. Schematic representation of time course analyses of the

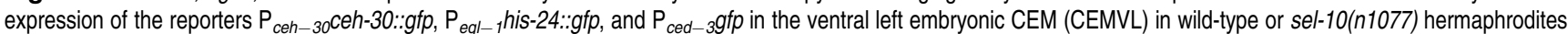
Time ( $\mathrm{min}$ ) is indicated on the left. Three embryos were analyzed for each reporter and genotype. White dots represent the CEM mother cells, green dots indicate CEMs expressing a particular gfp reporter. Green dots surrounded by purple circles indicate CEMs at the time when expression of the gfp reporter was first detected. (See Supplementary Figure S1 for corresponding DIC and fluorescent images.) Gray dots indicate CEMs lacking reporter expression. Embryos were prepared for 4D microscopy and lineaged starting at the 2- or 4-cell stage as described in Materials and Methods. Fluorescent stacks were taken every $\sim 20$ min after the CEM mother cell was born. No expression was detected for any of the reporters in the CEM mother cell

males as well as in masculinized hermaphrodites, indicating that ceh-30 is required for CEM survival ${ }^{13,14}(\mathrm{P}$ Grote and $\mathrm{B}$ Conradt, unpublished data). Using a translational ceh-30

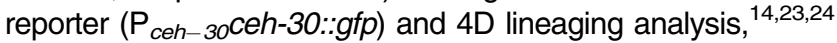
we determined the expression pattern of the ceh-30 gene in the CEMs in embryos starting at $\sim 320 \mathrm{~min}$, the time at which they are generated, until $\sim 470 \mathrm{~min}$, the time at which the CEMs normally die in hermaphrodites. ${ }^{6}$ We found that in wild-type hermaphrodites, in which the CEMs die, ceh-30 expression in the CEMs can only be detected right after the cells are generated (Figure 2, +/+; Supplementary Figure S1). ceh-30 expression cannot be detected in the mother cell and can no longer be detected in the CEMs at $\sim 360$ min or later time points (Figure 2). In masculinized hermaphrodites, however, in which the CEMs survive, ceh-30 expression in the CEMs can be detected starting at $\sim 400 \mathrm{~min}$ (Figure 2, sel-10(n1077)).

Next, we analyzed the expression of the ceh-30 reporter in the CEMs in masculinized hermaphrodites lacking unc-86, Irs-1, or unc-132 function in which, as described above, the CEMs are generated and survive, but are misspecified. We found that in these animals, at $\sim 470 \mathrm{~min}$, ceh-30 is not expressed in most CEMs (Figures $3 a$ and $b$ ). This result was surprising as the loss of ceh-30 function causes the CEMs to inappropriately die in masculinized hermaphrodites. On the basis of these observations, we conclude that unc-86, Irs-1, and unc-132 are required for the expression of ceh-30 in the
CEMs in masculinized hermaphrodites (Figure 4). We also conclude that apart from promoting ceh-30 expression, unc-86, Irs-1, and unc-132 have an additional function, which is to promote CEM death in a ceh-30-independent manner (Figure 4).

The loss of unc-86, Irs-1, or unc-132 function causes de-repression of egl-1 transcription in the CEMs in masculinized hermaphrodites. Using a transcriptional reporter $\left(\mathrm{P}_{\text {egl-1}}\right.$ his-24::gfp) and 4D lineaging analysis, we analyzed the expression of the egl-1 gene in the CEMs. ${ }^{25} \mathrm{We}$ found that in wild-type hermaphrodites, in which the CEMs die, egl-1 transcription can be detected in the CEMs starting at $\sim 400$ min (Figure 2, $+/+$; Supplementary Figure S1). In contrast, in masculinized hermaphrodites, in which the CEMs survive, egl-1 transcription cannot be detected in the CEMs (Figure 2, sel-10(n1077)). As mentioned above, the loss of ceh-30 function causes the CEMs to inappropriately die in masculinized hermaphrodites. We found that the loss of ceh-30 function causes egl-1 to be inappropriately transcribed in the CEMs in masculinized hermaphrodites at $\sim 470 \mathrm{~min}$ (Figures $5 \mathrm{a}$ and b). These results show that ceh-30 functions to repress egl-1 transcription in the CEMs in masculinized hermaphrodites (Figure 4).

To determine why the CEMs in masculinized hermaphrodites lacking unc-86, Irs-1, or unc-132 function, but not ceh-30 
a

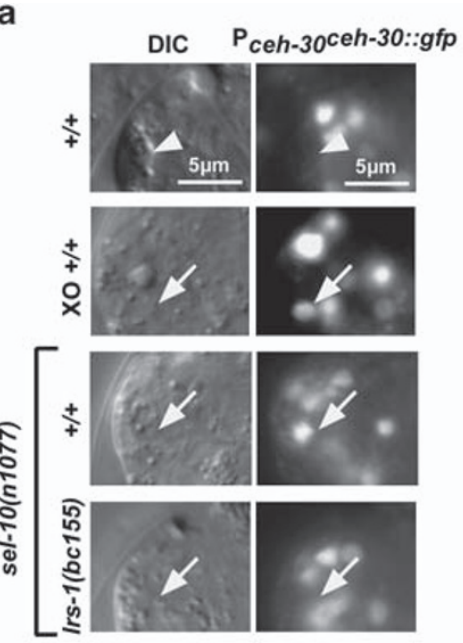

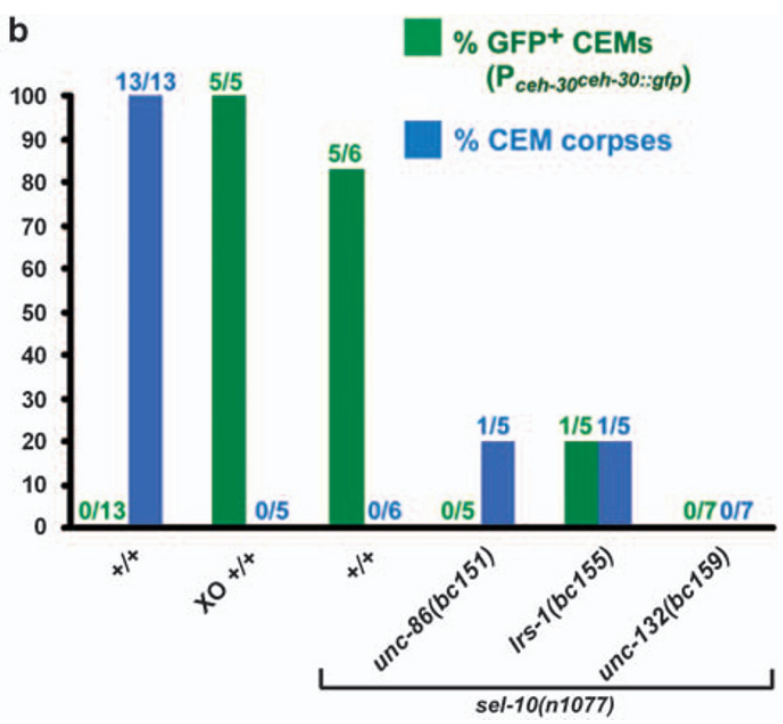

Figure 3 unc-86, Irs-1, and unc-132 are required for ceh-30 expression in the CEMs in masculinized hermaphrodites. (a) DIC and fluorescence images $\left(\mathrm{P}_{\text {ceh-30ceh- }}\right.$ 30::gfp) of CEM corpses (white arrow heads) or CEMs (white arrows) in wild-type hermaphrodites (+ I + ), males (XO + I + ), sel-10(n1077) hermaphrodites or Irs-1 (bc155); sel-10(n1077) hermaphrodites. Males were identified by recovering embryos after analysis and allowing them to develop into larvae. (b) Summary of data obtained on $\mathrm{P}_{\text {ceh-30 }}$ Ceh-30::gfp expression. Green bars indicate the percentage of CEMs that were GFP-positive and green numbers above indicate the fraction of CEMs analyzed that were GFP-positive. Blue bars indicate the percentage of CEMs that acquired a corpse-like morphology and blue numbers above indicate the fraction of CEMs analyzed that had a corpse-like morphology. All strains analyzed were homozygous for unc-76(e911) and carried the extrachromosomal array $n E x 1171$ ( $\left.P_{c e h-30} c e h-30:: g f p\right)$. The wild-type hermaphrodites and males analyzed were homozygous for him-5(e1467ts). The strains unc-86(bc151); sel-10(n1077) and Irs-1(bc155); sel-10(n1077) were also homozygous for $d p y-17(e 164)$

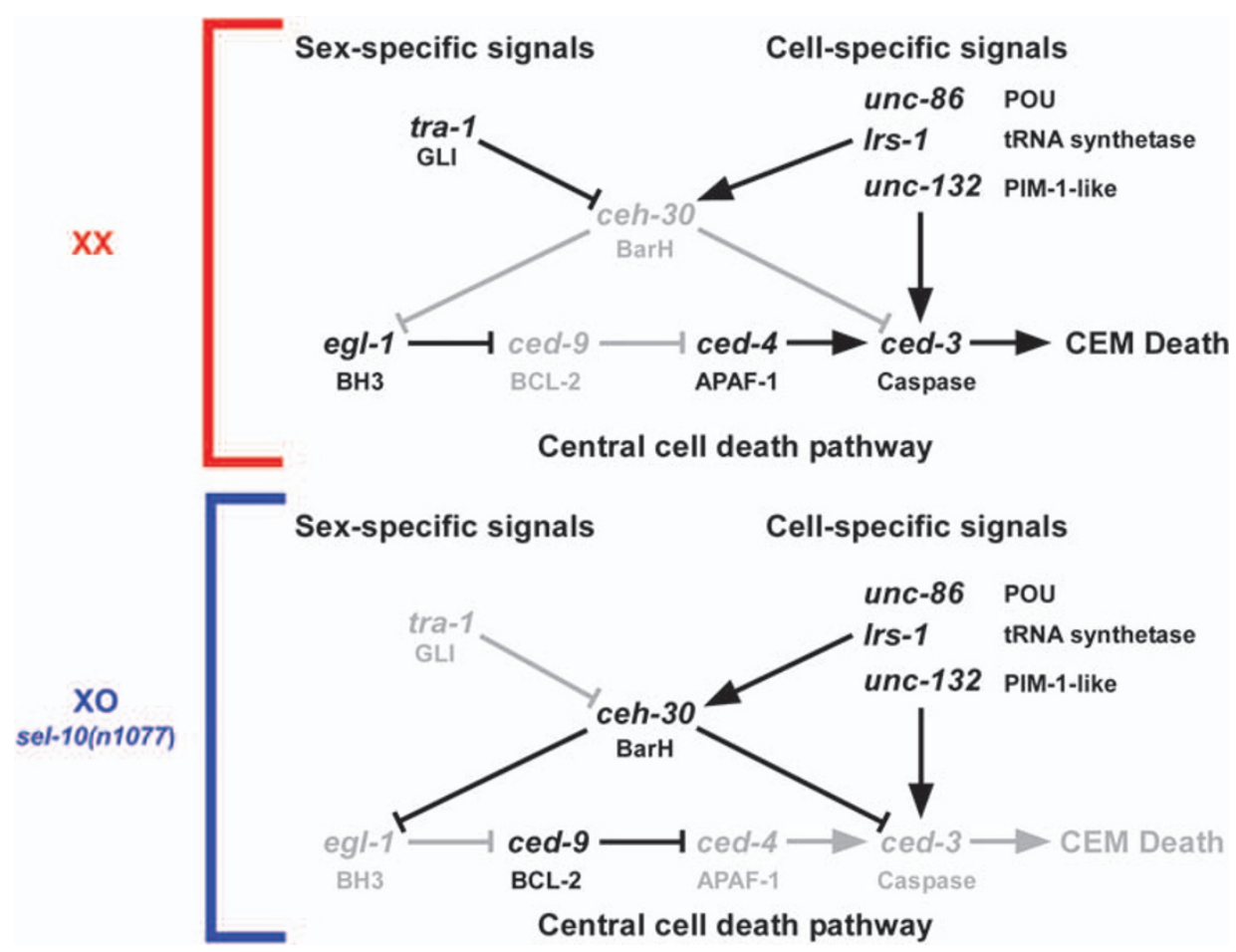

Figure 4 Genetic pathway of the CEM death. (Upper panel) In wild-type hermaphrodites (XX), tra-1 is active in the CEMs, thereby blocking ceh-30. Thus, the pro-apoptotic genes egl-1 and ced-3are active, which promotes CEM death. See text for details. (Lower panel) In males (XO) or masculinized hermaphrodites (sel-10(n1077)), tra-1 is not active in the CEMs, which results in the activation of ceh-30, and, consequently, the repression of egl-1 and ced-3. Thus, CEM death is inhibited. See text for details 
a b
$\% \mathrm{GFP}^{+}$CEMs

(Pegl-1his-24::gfp)

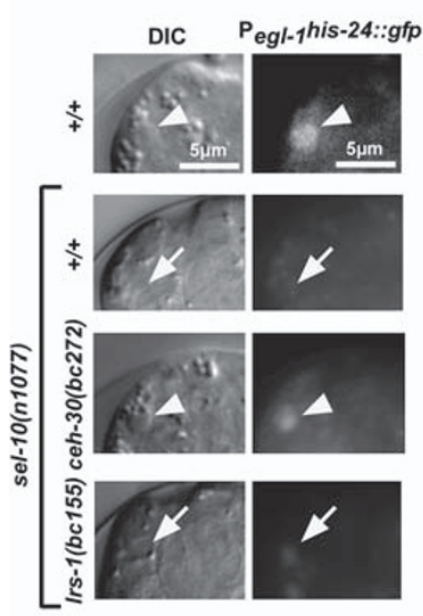

$\%$ CEM corpses

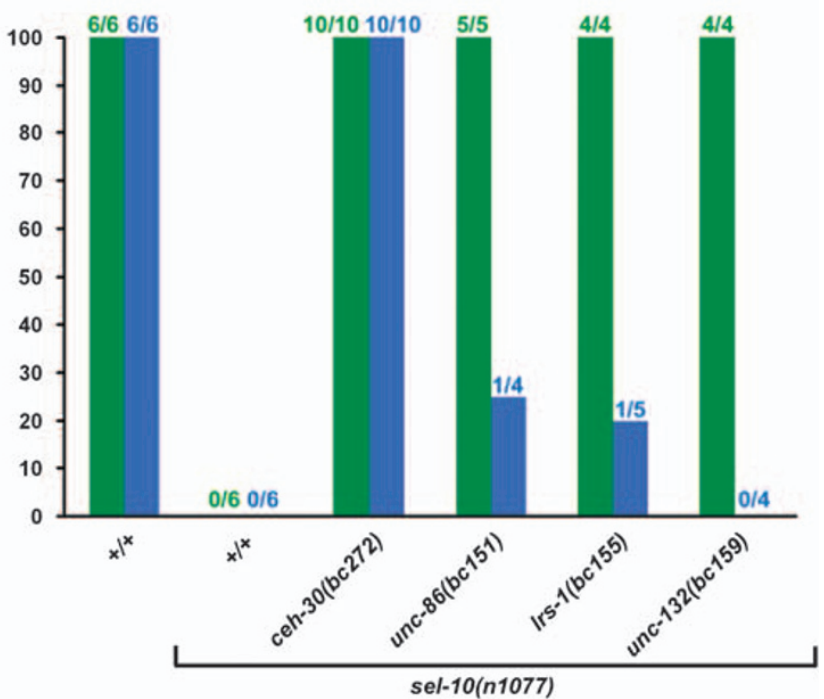

Figure 5 egl-1 is transcribed in surviving CEMs in masculinized unc-86(bc151), Irs-1(bc155), or unc-132(bc159) hermaphrodites. (a) DIC and fluorescence images ( $\mathrm{P}_{\text {egl-1 }}$ his-24::-gfp) of CEMs (white arrows) or CEM corpses (white arrow heads) in wild-type hermaphrodites (+ I + ), sel-10(n1077), sel-10(n1077); ceh-30(bc272), or Irs1(bc155); sel-10(n1077) hermaphrodites. (b) Summary of data obtained on $\mathrm{P}_{\text {egl-1 }}$ his-24:::gfp expression. Green bars indicate the percentage of CEMs that were GFP-positive and blue bars indicate the percentage of CEMs that acquired a corpse-like morphology. All strains analyzed were homozygous for the integrated $P_{e g l-1}$ his-24::gfp array bcls37. The strain ceh-30(bc272); sel-10(n1077) was also homozygous for the integrated array bcls9. The strains unc-86(bc151); sel-10(n1077) and Irs-1(bc155); sel-10(n1077) were homozygous for dpy-17(e164)

function, fail to inappropriately die, we analyzed egl-1 transcription in the CEMs in masculinized hermaphrodites lacking unc-86, Irs-1, or unc-132 at $\sim 470 \mathrm{~min}$. To our surprise, we found that the CEMs in these animals transcribe egl-1 at this time point (Figures $5 \mathrm{a}$ and b). This observation indicates that, unlike in masculinized hermaphrodites lacking ceh-30 function, in masculinized hermaphrodites lacking unc-86, Irs-1, or unc-132 function, the transcriptional upregulation of egl-1 in the CEMs is not sufficient for their apoptotic death.

ced-3 is transcribed in the CEMs in hermaphrodites and its transcription in the CEMs in masculinized hermaphrodites is repressed by ceh-30. Using a reporter for the caspase gene ced-3, it has been shown that ced-3 is not transcribed in most of the 131 cells that are programmed to die. ${ }^{4}$ However, ced-3 is actively transcribed in at least one cell that is programmed to die, the tail spike cell. ${ }^{4}$ Like the CEMs, the tail spike cell survives much longer than most cells programmed to die $(\sim 300 \mathrm{~min})$ before it dies. For this reason, we explored whether the ced-3 gene is transcribed in the CEMs before their deaths in hermaphrodites.

Using a transcriptional reporter $\left(\mathrm{P}_{c e d-3} g f p\right)$ and $4 \mathrm{D}$ lineaging analysis, we found that in wild-type hermaphrodites, in which the CEMs die, ced-3transcription can be detected in the CEMs starting at $\sim 460 \mathrm{~min}$ (Figure $2,+/+$; Supplementary Figure S1). Therefore, the ced-3 gene is transcriptionally active in the CEMs. Furthermore, we found that in masculinized hermaphrodites, in which the CEMs survive, ced-3 transcription cannot be detected (Figure 2, sel-10(n1077)). Next, we determined the role of ceh-30 in ced-3 transcription in the CEMs. We found that in masculinized hermaphrodites lacking ceh-30 function, in which the CEMs inappropriately die, ced-3 is transcribed in the CEMs at $\sim 470 \mathrm{~min}$ (Figures $6 \mathrm{a}$ and $b$ ). Therefore, we conclude that ceh-30 functions to repress ced-3 transcription in the CEMs in masculinized hermaphrodites.

The loss of unc-86, Irs-1, or unc-132 function does not cause activation of ced-3 transcription in the CEMs in masculinized hermaphrodites. To determine the role of unc-86, Irs-1, and unc-132 in ced-3 transcriptional control in the CEMs, we analyzed the expression of the $\mathrm{P}_{c e d-3}$ gfp reporter in masculinized hermaphrodites lacking unc-86, Irs-1, or unc-132 function at $\sim 470 \mathrm{~min}$. We found that, like in masculinized hermaphrodites, the ced-3 gene is not transcribed in most CEMs at this time point (Figures $6 \mathrm{a}$ and $b$ ). Therefore, while the loss of unc-86, Irs-1, or unc-132 function results in the de-repression of egl-1 transcription in the CEMs in masculinized hermaphrodites, it does not result in the de-repression of ced-3 transcription. On the basis of this observation we conclude that unc-86, Irs-1, and unc-132 not only repress ced-3 transcription in a ceh-30-dependent manner but also promote ced-3 transcription in a ceh-30independent manner (Figure 4). Furthermore, we propose that the CEMs in masculinized hermaphrodites lacking unc-86, Irs-1, or unc-132 function fail to die because ced-3 transcription in the CEMs is not activated. 
a
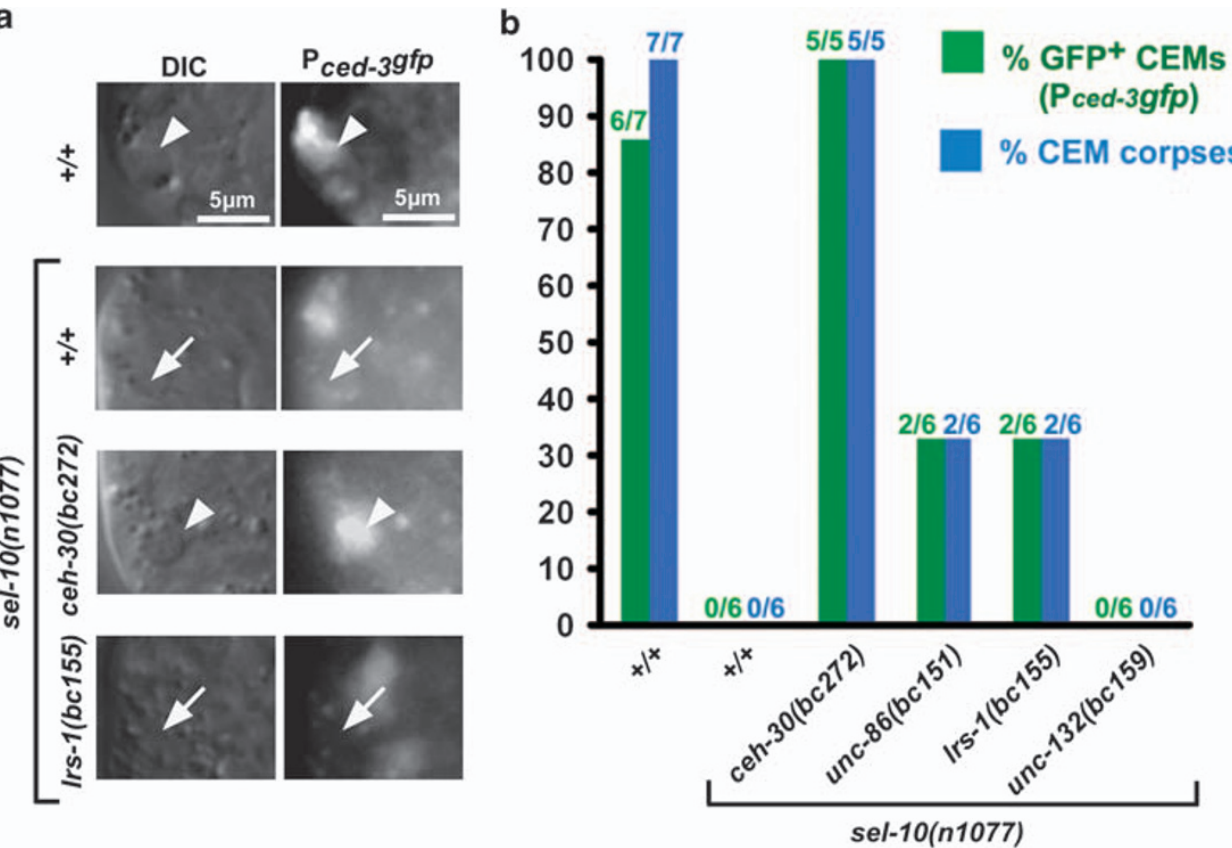

Figure 6 Analysis of ced-3 transcription in the CEMs. (a) DIC and fluorescence images ( $\mathrm{P}_{\text {ced-3 }} g f p$ ) of CEM corpses (white arrow heads) or CEMs (white arrows) in wildtype (+ I+ ), sel-10(n1077), sel-10(n1077); ceh-30(bc272), or Irs-1(bc155); sel-10(n1077) hermaphrodites. (b) Summary of data obtained on $\mathrm{P}_{\text {ced-3 }}$ gfp expression. Green bars indicate the percentage of CEMs that were GFP-positive, and blue bars indicate the percentage of CEMs that acquired a corpse-like morphology. All strains analyzed were homozygous for the integrated $\mathrm{P}_{p k d-2} g f p$ array bcls9 and carried extrachromosomal arrays of $\mathrm{P}_{c e d-3} g f p(+1+[b c E x 834, b c E X 836]$, sel-10(n1077) [bcEx839, bcEx840], ceh-30(bc272); sel-10(n1077) [bcEx876], unc-86(bc151); sel-10(n1077) [bcEx870, bcEx871], Irs-1(bc155); sel-10(n1077) [bcEx872, bcEx873], unc-132(bc159); sel-10(n1077) [bcEx874, bcEx875])

\section{Discussion}

Transcriptional upregulation of both egl-1 and ced-3 is required for the apoptotic death of the CEMs in hermaphrodites. The transcriptional upregulation of egl-1 is thought to be necessary and sufficient for many of the cell death events that take place during $C$. elegans development. $^{7}$ We found that egl-1 is transcriptionally active in the CEMs in hermaphrodites in which the CEMs die but not in masculinized hermaphrodites (and most probably males) in which the CEMs survive. However, our results show that the transcriptional upregulation of egl-1 in the CEMs in hermaphrodites is necessary, but not sufficient for their death.

The ced-3 gene does not appear to be transcriptionally active in many of the cells that are programmed to die during development. ${ }^{4}$ However, we found that $c e d-3$ is transcriptionally active in the CEMs in hermaphrodites in which the CEMs die but not in masculinized hermaphrodites in which the CEMs survive. Furthermore, we present data that indicate that transcriptional activation of ced-3 in the CEMs is necessary for their death in hermaphrodites. On the basis of these findings, we propose that in contrast to most cells that are programmed to die during development, in the CEMs, the transcriptional upregulation of egl-1 and ced-3 is necessary for their death in hermaphrodites. This is the first demonstration that the transcriptional upregulation of both a $\mathrm{BH} 3$-only gene as well as a caspase gene is required for apoptosis induction.

What distinguishes the CEMs from the majority of cells that die during development is that rather than surviving for only $\sim 30$ min after being generated, they survive for $\sim 150 \mathrm{~min}^{6}{ }^{6} \mathrm{In}$ the majority of cells that are programmed to die proCED-3 protein is inherited from progenitors. ${ }^{4}$ On the basis of this observation, we propose that at the time apoptosis induction takes place in the CEMs, as a result of proCED-3 turn over, the level of progenitor-derived proCED-3 has reached a level in the CEMs that is no longer sufficient for apoptosis induction (Figure 7). For this reason, the transcriptional upregulation of not only the egl-1 gene but also the ced-3 gene is necessary for apoptosis induction in the CEMs.

CEH-30 represses egl-1 and ced-3 transcription thereby promoting CEM survival. The inappropriate death of CEMs in males lacking ceh-30 function is suppressed by the loss of egl-1 function. ${ }^{14}$ This suggests that the egl-1 gene acts downstream of the ceh-30 gene and is a potential target of the $\mathrm{CEH}-30$ transcription factor. However, analyses performed with a gain-of-function mutation of ceh-30 suggest that the ceh-30 gene blocks the death of the CEMs by acting in parallel to or downstream of egl-1. ${ }^{14}$ We present data that indicate that the inappropriate death of CEMs in masculinized hermaphrodites lacking ceh-30 function is the result of the transcriptional de-repression of the pro-apoptotic genes egl-1 and ced-3. In other words, we show that the ceh-30 gene acts both upstream of as well as in parallel to egl-1 to prevent the activation of the central cell death machinery. We propose that the $\mathrm{CEH}-30$ protein is required to repress egl-1 and ced-3 transcription in the CEMs of masculinized hermaphrodites, thereby causing their survival 
Most cells programmed to die

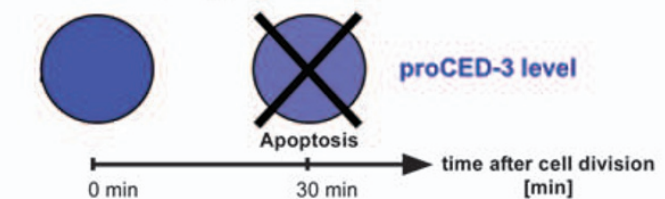

egl-1 TRANSCRIPTION
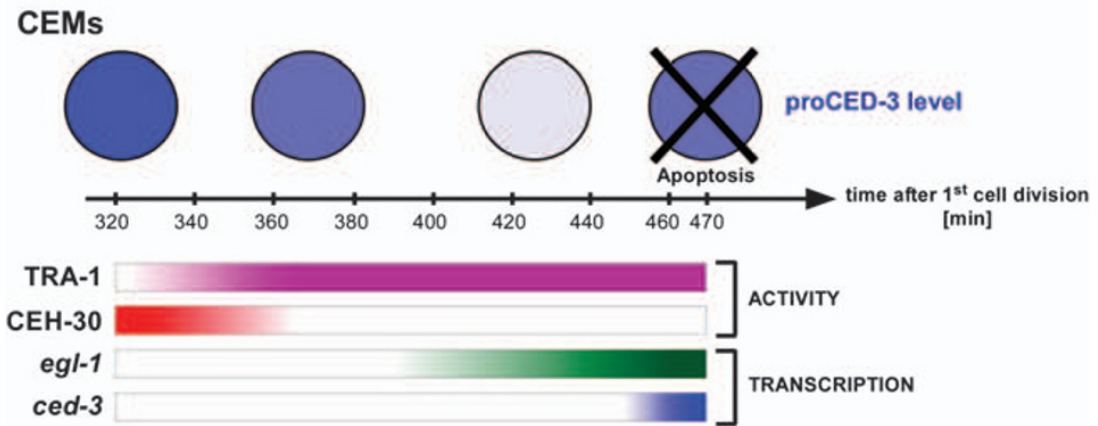

Figure 7 Model of the life-versus-death decision in the CEMs. (Top panel) Most cells programmed to die do so $\sim 30$ min after being generated. In these cells, proCED-3 protein inherited from the progenitor is sufficient for apoptosis induction in response to egl-1 transcriptional activation. (Bottom panel) The CEMs in hermaphrodites die $\sim 150$ min after being generated ( $\sim 470$ min after the first cell division). Increasing TRA-1 activity results in decreasing CEH-30 activity, which is required for the de-repression of egl-1 and ced-3 transcription. The activation at the transcriptional level of the ced-3 gene compensates for decreased levels of proCED-3. See text for details

(Figure 4). Whether $\mathrm{CEH}-30$ represses egl-1 and ced-3 transcription directly or indirectly remains to be determined.

ceh-30 transcription in the CEMs is dependent on unc-86, Irs-1, and unc-132 function. The ceh-30 gene is transcriptionally active in the CEMs in males and masculinized hermaphrodites, but not in hermaphrodites. In hermaphrodites, ceh-30 transcription in the CEMs is directly repressed by the GLI-like transcription factor TRA-1, which is the terminal, global regulator of somatic sexual fate ${ }^{12-14}$ (Figure 4). Our results indicate that the genes unc-86, Irs-1, and unc-132, which are required for correct CEM specification, are necessary for ceh-30 transcriptional activation in the CEMs in masculinized hermaphrodites (Figure 4).

unc-86 encodes a POU homeodomain transcription factor that has previously been implicated in CEM specification and differentiation. ${ }^{18,19}$ Our results show that the UNC-86 protein is required for ceh-30 transcription in the CEMs, which is consistent with previous studies that suggest that UNC-86 acts as a direct activator of ceh-30 transcription. ${ }^{13}$ Surprisingly, Irs-1 encodes a leucyl-tRNA synthetase. On the basis of our analyses, compromising translation by decreasing the level of at least certain aminoacyl-tRNA synthetases abrogates ceh-30 transcription in the CEMs. Therefore, we propose that ceh-30 transcription is particularly sensitive to perturbations of the translational machinery. Finally, unc-132 encodes a novel protein of unknown function. The $D$. melanogaster orthologue of unc-132, CG30118, was identified in a screen for genes involved in Drosophila hematopoiesis. ${ }^{21}$ However, the mechanism through which CG30118 acts has so far not been determined. Furthermore, we found that the $\mathrm{N}$-terminal region of the UNC-132 protein shares sequence similarity with the $\mathrm{N}$-terminal region of the human protooncoprotein PIM-1. ${ }^{22} \mathrm{PIM}-1$ is a serine/threonine kinase, which is involved in various cellular processes. ${ }^{26}$ However, the roles of PIM-1 in these processes are thought to be dependent on its kinase activity. As UNC-132 does not contain a classical kinase domain, it is unclear how extensive the functional similarities between PIM-1 and UNC-132 are. In summary, although UNC-86 may be a direct activator of ceh30 transcription, the mechanisms through which LRS-1 and UNC-132 promote ceh-30 transcription in the CEMs remain to be elucidated.

unc-86, Irs-1, and unc-132 promote ced-3 transcription in the CEMs in a ceh-30-independent manner thereby promoting their death. unc-86, Irs-1, and unc-132 are required for ceh-30 transcriptional activation in the CEMs in masculinized hermaphrodites. However, although we found that the loss of ceh-30 function results in the de-repression of both egl-1 and ced-3 transcription, the loss of unc-86, Irs-1, or unc-132 function only results in the activation of egl-1 transcription. For this reason, we propose that apart from blocking egl-1 and ced-3 transcription in a ceh-30-dependent manner, unc-86, Irs-1, and unc-132 also promote ced-3 transcription in a ceh-30-independent manner (Figure 4). We hypothesize that the ability of unc-86, Irs-1, and unc-132 to promote ced-3 transcription in a ceh-30-independent manner is relevant in hermaphrodites in which the ced-3 gene is relieved of ceh-30-dependent transcriptional repression (Figure 4, XX). Conversely, their ability to block ced-3 transcription in a ceh-30-dependent manner may be relevant in males and masculinized hermaphrodites in which the ceh-30 gene is relieved of TRA-1-dependent transcriptional repression (Figure 4, XO, sel-10(n1077)). How unc-86, Irs-1, and unc-132 promote ced-3 transcription in the CEMs in hermaphrodites remains to be determined. 
Why do the CEMs survive for $\mathbf{1 5 0} \mathbf{m i n}$ ? The CEMs are born $\sim 320 \mathrm{~min}$ after the first cell division and die in hermaphrodites at $\sim 470$ min. $^{6}$ The TRA-1 protein has a pivotal function in the life-versus-death decision of the CEMs. In the CEMs, TRA-1 represses ceh-30 transcription thereby causing egl-1 and ced-3 de-repression and apoptosis induction. The activity of TRA-1 is regulated at the posttranslational level by sex-specific cleavage and proteolysis. ${ }^{12}$ Currently, it is unclear when during embryonic development active TRA-1 protein is first generated in hermaphrodites. However, experiments using a temperature-sensitive lossof-function mutation of the gene tra-2, which acts upstream of tra-1 in somatic sex determination, suggest that active TRA-1 might be generated in hermaphrodites starting at $\sim 320$ min. ${ }^{27}$ This notion is supported by the finding that the ceh-30 gene, whose expression is directly repressed by TRA-1, is no longer expressed at $\sim 360$ min (Figures 2 and 7). On the basis of these observations, we propose that the CEMs die only at $\sim 470 \mathrm{~min}$, because a level of active TRA-1 first has to be generated in the CEMs in hermaphrodites that is sufficient for the transcriptional repression of ceh-30, which is a prerequisite for the transcriptional de-repression of egl-1 and ced-3 at $\sim 390 \mathrm{~min}$ and $460 \mathrm{~min}$, respectively (Figure 7). Hence, we propose that the timing of the death of the CEMs is controlled by the sex determination pathway and, in particular, its terminal, global regulator, the Gli-like transcription factor TRA-1.

Potential role for the coexpression of BH3-only and caspase genes in the developmental control of apoptosis. At least during animal development, pro-caspases are thought to be present in most if not all cells. ${ }^{1,2}$ Furthermore, pro-caspases are thought to be present at levels that, once they become matured and activated, are sufficient for apoptosis induction. As the maturation and activation of pro-caspases generally represents the commitment of a cell to the apoptotic fate, it is a highly regulated process. ${ }^{28}$ In contrast, very little is known thus far about the contribution of transcriptional control in the regulation of pro-caspases. The most comprehensive studies available to date were performed in $D$. melanogaster. These studies suggest that transcriptional regulation of caspase genes has an important function in apoptosis induction at least during D. melanogaster development. ${ }^{1,29}$ The data presented here show that the transcriptional upregulation of the $C$. elegans caspase gene ced-3 is required for the sexually dimorphic, apoptotic death of the CEM neurons. On the basis of these findings, we propose that the transcriptional upregulation of caspase genes is a conserved aspect of apoptosis induction during animal development. Furthermore, our data show that in $C$. elegans, genes encoding $\mathrm{BH} 3$-only proteins and pro-caspases can be under the same transcriptional control. We hypothesize that such coregulatory mechanisms have evolved to ensure efficient apoptosis induction during development.

\section{Materials and Methods}

Strains and general methods. $C$. elegans strains were cultured as described. ${ }^{30}$ Bristol N2 was used as the wild-type strain. N2 and the CB4856 (Hawaii) strain were used for SNP mapping. Mutations and integrated transgenes used in this study are listed below and are described ${ }^{31}$ except where noted otherwise: LG II: rrf-3(pk1426)..$^{32}$ LG III: ced-4(n1162), unc-86(bc151) (this study), Irs-1 (bc155) (this study), dpy-17(e164), unc-93(e1500sd). LG IV: ced-3(n717) LG V: bcls37 ( $P_{\text {egl- } 1}$ his-24::gfp), ${ }^{25}$ egl-1(n1084 n3082), ${ }^{10}$ sel-10(n1077), unc-132(bc159) (this study), unc-76(e911), him-5(e1490), him-5(e1467ts), dpy-11(e224). LG X: ceh-30(bc272) (P. Grote and B. Conradt; unpublished data), lin-15(n765ts), bcls9 $\left(\mathrm{P}_{p k d-2} g f p\right){ }^{33}$ The following extrachromosomal arrays were used:

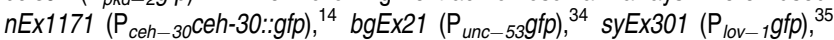
$n s E x 37\left(\mathrm{P}_{c f i-1} g f p\right),{ }^{36}$ akEx32 $\left(\mathrm{P}_{\text {glr }-4} g / r-4: .: g f p\right) .{ }^{37}$

RNAi by feeding was performed as described using 6 mM IPTG. ${ }^{38}$ The plasmids pBC966 (W08A12.1b(RNAi)) and pBC967 (W08A12.1C(RNAi)) contained full-length cDNA clones cloned as Ncol-Nhel fragments into vector L4440. sel-10(n1077); bcls9 animals were mutagenized with EMS (ethyl-methanesulfonate) as described. ${ }^{30}$ Germline transformations were performed as described. ${ }^{39}$ Cosmids and fosmids were injected at a concentration of $10 \mathrm{ng} / \mu \mathrm{l}$ using pRF4 (rol-6(su1006)) at $50 \mathrm{ng} / \mu \mathrm{l}$ as coinjection marker. The plasmid $\mathrm{P}_{c e d-3}$ gfp was injected at $40 \mathrm{ng} / \mu \mathrm{l}$ using pRF4 (rol-6(su1006)) $(50 \mathrm{ng} / \mu \mathrm{l})$ as coinjection marker. In the case of bcls 9 $\left(\mathrm{P}_{\text {pkd-2 }} g f p\right)$, syEx301 ( $\left.\mathrm{P}_{\text {lov-1 }} g f p\right)$, bgEx21 ( $\left.\mathrm{P}_{\text {unc-53 }} g f p\right), n s E x 37\left(\mathrm{P}_{\text {cfi- } 1}\right.$ gfp), akEx32 $\left(\mathrm{P}_{\text {glr-4 }}\right.$ glr-4:::gfp), bcls37 ( $\mathrm{P}_{\text {egl-1 }}$ his-24::gfp), and nEx1171 ( $\mathrm{P}_{\text {ceh-30 }}$ ceh-30::gfp), transgenic animals were crossed with unc-86(bc151), Irs-1(bc155) or unc132(bc159) mutants to generate unc-86(bc151), Irs-1(bc155) and unc-132(bc159) strains carrying these arrays. pBC957 ( $\left.\mathrm{P}_{c e d-3} g f p\right)$ was constructed by removing an internal, $4.6 \mathrm{~kb}$ EcoRV fragment containing most of the ced-3 coding sequence from a ced-3 reporter. ${ }^{4}$

sel-10(n1077) screen and cloning of unc-86(bc151), Irs-1(bc155), and unc-132(bc159). 'Undead' CEMs in sel-10(n1077) hermaphrodites have the characteristic CEM morphology when observed by differential interference contrast microscopy (DIC) in L4 larvae, and express CEM-specific markers in adults, such as the genes pkd-2 ( $\left.\mathrm{P}_{p k d-2} g f p\right)$ and lov-1 $\left(\mathrm{P}_{\text {lov-1 }} g f p\right){ }^{35}$ These observations indicate that the CEMs in sel-10(n1077) hermaphrodites are correctly specified and fully differentiated. We mutagenized sel-10(n1077) hermaphrodites carrying an integrated $\mathrm{P}_{p k d-2} g f p$ transgene $(b c / s 9)$, screened 8500 mutagenized, haploid genomes for mutants in which differentiated CEMs were absent based on pkd-2 expression and identified 49 mutants. Among these mutants, 21 still had CEMs based on DIC and therefore most likely harbor mutations that affect pkd-2 expression. In addition, in 20 mutants, all aspects of the weak masculinization caused by sel-10(n1077) were suppressed, indicating that they most likely carry feminizing mutations or loss-of-function mutations in the sel-10 gene. Of the remaining eight mutations, one behaved non-Mendelian, one could not be outcrossed, and three caused the CEMs to be absent in masculinized sel-10(n1077) hermaphrodites, but not males. The final three mutations, bc151, bc155, and bc159, were characterized further.

Standard genetic techniques were used to map $b c 151$ to the right of $d p y-17$ on LG III, bc155 between unc-93 and dpy-17 on LG III, and bc159 to the left of dpy-11 on LG V.

$b c 151: b c 151$ failed to complement unc-86(n846), a If allele of the gene unc-86, indicating that $b c 151$ is a loss-of-function mutation in the unc-86 gene.

bc155:SNP mapping was used to locate bc155 between the SNPs C30D 11:9408 and R10E4:10469. The fosmid WRM0638-J8 rescued the phenotype observed in bc155; sel-10(n1077); bcls9 animals.

$b c 159: S N P$ mapping was used to map bc159 to the left of SNP H10D18:26865. The cosmid W08A12 rescued the phenotype observed in bc159; sel-10(n1077); $b c / s 9$ animals. The $b c 159$ phenotype was also rescued by a $31.3-\mathrm{kb} \mathrm{Xcml}$ subclone of W08A12 (pBC430, containing the genes W08A12.1, W08A12.2, and egr-1) and was partially rescued by a 23.9-kb Apal subclone of W08A12 (pBC431, spanning the genes W08A12.4, egr-1, and W08A12.1).

Phenotypic analysis. The presence of CEMs in adults was analyzed using $b c / s 9\left(\mathrm{P}_{p k d-2} g f p\right)$ and a Zeiss Axioskop2 equipped with epifluorescence as described. ${ }^{33}$ The \% GFP-positive CEMs was calculated by dividing the number of CEMs observed by the maximum number of possible CEMs (four CEMs per animal). The presence of CEMs in $\mathrm{L} 4$ larvae was analyzed using DIC as described. ${ }^{8}$ The presence of CEMs in embryos was analyzed as follows. CEMs were identified by DIC in $1 \frac{1}{2}$-fold stage embryos ( $450 \mathrm{~min}$ ) based on their positions and observed for at least $\sim 30 \mathrm{~min}$. (As the positions of the two dorsal CEMs are less characteristic, these cells could not always be examined.) CEM corpses appear in two-fold embryos ( $465 \mathrm{~min}$ ). Microscopy of living embryos was performed by mounting embryos on $2 \%$ agar pads in M9 buffer, using a Zeiss Axioskop2 
equipped with epifluorescence, a Micromax CCD camera (Princeton Instruments, Trenton, NJ, USA), and Metamorph software. DIC and epifluorescence images were taken every 5 min between the $1 \frac{1}{2}$-and 2 -fold stage to determine the fate of the CEMs and the expression of the GFP reporters in embryonic CEMs.

The presence of the AMso (amphid socket) cells was scored in adults using $P_{\text {unc-53 }}$ gfp as described. ${ }^{37}$ The presence of the URA cells was determined in adults using two different reporters, $\mathrm{P}_{\text {glr- }-4} g r l-4: .: g f p$ and $\mathrm{P}_{\text {cfi- } 1}$ gfp, as described. ${ }^{36}$

4D microscopy and lineage analysis. 4D microscopy and lineaging analysis (as shown in Figure 2) was performed on $C$. elegans embryos as described earlier. ${ }^{23,24}$ Embryos were recorded at $20^{\circ} \mathrm{C}$, and GFP expression of the reporters

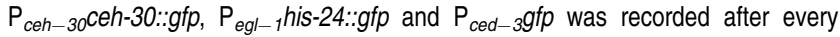
$30 \mathrm{DIC}$ stacks. The 4D recordings were analyzed using the SIMI BioCell software as described earlier. ${ }^{23,40}$ The ventral left CEM (CEMVL, ABplpaapapp) was lineaged in all embryos. Starting at the 2- to 4-cell stage, cells were tracked and their $3 \mathrm{D}$ coordinates were saved approximately every $2 \mathrm{~min}$, until at least $\sim 465 \mathrm{~min}$ after the first cell division. In addition, the $3 \mathrm{D}$ coordinates of the CEMVL progenitor (ABplpaapap) or CEMVL were saved at the time points when GFP expression of the

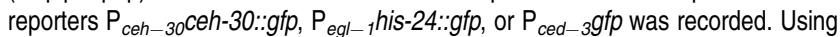
this approach, the status of expression of these reporters in the CEM could be determined. The cell death fate of the CEMVL was determined at $\sim 465 \mathrm{~min}$ by DIC.

To determine the expression of the reporters $\mathrm{P}_{\text {ceh-30 }}$ ceh-30::gfp, $\mathrm{P}_{\text {egl- } 1}$ his24.:.gfp, or $\mathrm{P}_{\text {ced }-3} g f p$ at $\sim 470 \mathrm{~min}$ after the first cell division (as shown in Figures 3 , 5 , and 6), we identified the CEMs in embryos $\sim 450$ min after the first cell division based on their positions using DIC and observed them for $\sim 30 \mathrm{~min}$ using fluorescence microscopy. At the end of the recording, we determined the cell death fate of the CEMs using DIC (Apoptotic cells adopt a button-like, refractile appearance, which can be detected by DIC).

\section{Conflict of interest}

The authors declare no conflict of interest.

Acknowledgements. We thank E. Lambie and S. Rolland for comments on the manuscript; H. Schwartz, M. Saito, J. Dunlap, and members of the Conradt laboratory for discussions; D. Mayka and C. Huber for excellent technical support; $E$. Lambie for use of the micro-injection set-up; H. Schwartz and B. Horvitz for array $n E x 1171\left(\mathrm{P}_{\text {ceh-30 }}\right.$ ceh-30::gfp); S. Shaham for array nsEx37 ( $\mathrm{P}_{c f i-1}$ gfp) and a ced-3 reporter; N. Pujol for array bgEx21 ( $\left.\mathrm{P}_{\text {unc-53 }} g f p\right)$; V. Maricq for array akEx32 $\left(\mathrm{P}_{\text {glr }-4} g / \mathrm{l}-4: .: g f p\right) ;$ M. Barr for array syEx301 ( $\left.\mathrm{P}_{\text {lov }-1} g f p\right)$ and plasmid $\mathrm{P}_{p k d-2} g f p$; the Sanger Centre (Hinxton, UK) for cosmids; and the C. elegans Genetics Center (CGC, supported by the NIH National Center for Research Resources) for strains. This work was supported by funding from the Max Planck Society, Howard Hughes Medical Institute Award 76200-560801 to Dartmouth Medical School under the Biomedical Research Support Program for Medical Schools, and National Institute of Health grant R01-GM069950.

1. Conradt B. Genetic control of programmed cell death during animal development. Annu Rev Genet 2009; 43: 493-523.

2. Horvitz HR. Nobel lecture. Worms, life and death. Biosci Rep 2003; 23: 239-303.

3. Chen F, Hersh BM, Conradt B, Zhou Z, Riemer D, Gruenbaum Y et al. Translocation of C. elegans CED-4 to nuclear membranes during programmed cell death. Science 2000; 287: $1485-1489$.

4. Maurer CW, Chiorazzi M, Shaham S. Timing of the onset of a developmental cell death is controlled by transcriptional induction of the $C$. elegans ced-3 caspase-encoding gene. Development 2007; 134: 1357-1368.

5. Shaham S, Horvitz HR. Developing Caenorhabditis elegans neurons may contain both cell-death protective and killer activities. Genes Dev 1996; 10: 578-591.

6. Sulston JE, Schierenberg E, White JG, Thomson JN. The embryonic cell lineage of the nematode Caenorhabditis elegans. Dev Biol 1983; 100: 64-119.

7. Nehme R, Conradt B. egl-1: a key activator of apoptotic cell death in C. elegans. Oncogene 2008; 27 (Suppl 1): S30-S40.

8. Sulston JE, Horvitz HR. Post-embryonic cell lineages of the nematode, Caenorhabditis elegans. Dev Biol 1977; 56: 110-156.

9. White JQ, Nicholas TJ, Gritton J, Truong L, Davidson ER, Jorgensen EM. The sensory circuitry for sexual attraction in C. elegans males. Curr Biol 2007; 17: 1847-1857.
10. Conradt B, Horvitz HR. The $C$. elegans protein EGL-1 is required for programmed cell death and interacts with the Bcl-2-like protein CED-9. Cell 1998; 93: 519-529.

11. Ellis HM, Horvitz HR. Genetic control of programmed cell death in the nematode C. elegans. Cell 1986; 44: 817-829.

12. Wolff JR, Zarkower D. Chapter 1 somatic sexual differentiation in Caenorhabditis elegans. Curr Top Dev Biol 2008; 83: 1-39.

13. Peden E, Kimberly E, Gengyo-Ando K, Mitani S, Xue D. Control of sex-specific apoptosis in C. elegans by the BarH homeodomain protein $\mathrm{CEH}-30$ and the transcriptional repressor UNC-37/Groucho. Genes Dev 2007; 21: 3195-3207.

14. Schwartz HT, Horvitz HR. The $C$. elegans protein $\mathrm{CEH}-30$ protects male-specific neurons from apoptosis independently of the Bcl-2 homolog CED-9. Genes Dev 2007; 21: 3181-3194.

15. Desai C, Horvitz HR. Caenorhabditis elegans mutants defective in the functioning of the motor neurons responsible for egg laying. Genetics 1989; 121: 703-721.

16. Doniach T. Activity of the sex-determining gene tra-2 is modulated to allow spermatogenesis in the C. elegans hermaphrodite. Genetics 1986; 114: 53-76.

17. Jager S, Schwartz HT, Horvitz HR, Conradt B. The Caenorhabditis elegans F-box protein SEL-10 promotes female development and may target FEM-1 and FEM-3 for degradation by the proteasome. Proc Natl Acad Sci USA 2004; 101: 12549-12554.

18. Finney $M$, Ruvkun $G$. The unc- 86 gene product couples cell lineage and cell identity in C. elegans. Cell 1990; 63: 895-905.

19. Finney M, Ruvkun G, Horvitz HR. The $C$. elegans cell lineage and differentiation gene unc-86 encodes a protein with a homeodomain and extended similarity to transcription factors. Cell 1988; 55: 757-769.

20. Schimmel P. Development of tRNA synthetases and connection to genetic code and disease. Protein Sci 2008; 17: 1643-1652.

21. Milchanowski AB, Henkenius AL, Narayanan M, Hartenstein V, Banerjee U. Identification and characterization of genes involved in embryonic crystal cell formation during Drosophila hematopoiesis. Genetics 2004; 168: 325-339.

22. Nagarajan L, Louie E, Tsujimoto Y, ar-Rushdi A, Huebner K, Croce CM. Localization of the human pim oncogene (PIM) to a region of chromosome 6 involved in translocations in acute leukemias. Proc Natl Acad Sci USA 1986; 83: 2556-2560.

23. Bischoff M, Schnabel R. A posterior centre establishes and maintains polarity of the Caenorhabditis elegans embryo by a Wnt-dependent relay mechanism. PLoS Biol 2006; 4: e396.

24. Schnabel R, Hutter $H$, Moerman D, Schnabel $H$. Assessing normal embryogenesis in Caenorhabditis elegans using a 4D microscope: variability of development and regional specification. Dev Biol 1997; 184: 234-265.

25. Thellmann M, Hatzold J, Conradt B. The Snail-like CES-1 protein of $C$. elegans can block the expression of the $\mathrm{BH} 3$-only cell-death activator gene egl-1 by antagonizing the function of bHLH proteins. Development 2003; 130: 4057-4071.

26. Bachmann M, Moroy T. The serine/threonine kinase Pim-1. Int J Biochem Cell Biol 2005; 37: 726-730.

27. Klass $M$, Wolf $N$, Hirsh $D$. Development of the male reproductive system and sexual transformation in the nematode Caenorhabditis elegans. Dev Biol 1976; 52: 1-18.

28. Schafer ZT, Kornbluth S. The apoptosome: physiological, developmental, and pathological modes of regulation. Dev Cell 2006; 10: 549-561.

29. Kumar S, Cakouros D. Transcriptional control of the core cell-death machinery. Trends Biochem Sci 2004; 29: 193-199.

30. Brenner S. The genetics of Caenorhabditis elegans. Genetics 1974; 77: 71-94.

31. Riddle DL. C. elegans II. Cold Spring Harbor Laboratory Press: Cold Spring Harbor, 1997.

32. Simmer F, Tijsterman M, Parrish S, Koushika SP, Nonet ML, Fire A et al. Loss of the putative RNA-directed RNA polymerase RRF-3 makes $C$. elegans hypersensitive to RNAi. Curr Biol 2002; 12: 1317-1319.

33. Grote P, Conradt B. The PLZF-like protein TRA-4 cooperates with the Gli-like transcription factor TRA-1 to promote female development in C. elegans. Dev Cell 2006; 11: 561-573.

34. Stringham E, Pujol N, Vandekerckhove J, Bogaert T. unc-53 controls longitudinal migration in C. elegans. Development 2002; 129: 3367-3379.

35. Barr MM, Sternberg PW. A polycystic kidney-disease gene homologue required for male mating behaviour in C. elegans. Nature 1999; 401: 386-389.

36. Shaham $\mathrm{S}$, Bargmann $\mathrm{Cl}$. Control of neuronal subtype identity by the $\mathrm{C}$. elegans ARID protein CFI-1. Genes Dev 2002; 16: 972-983.

37. Brockie PJ, Madsen DM, Zheng Y, Mellem J, Maricq AV. Differential expression of glutamate receptor subunits in the nervous system of Caenorhabditis elegans and their regulation by the homeodomain protein UNC-42. J Neurosci 2001; 21: 1510-1522.

38. Timmons $\mathrm{L}$, Court DL, Fire A. Ingestion of bacterially expressed dsRNAs can produce specific and potent genetic interference in Caenorhabditis elegans. Gene 2001; 263: 103-112.

39. Mello C, Fire A. DNA transformation. Methods Cell Biol 1995; 48: 451-482.

40. Schnabel R, Bischoff M, Hintze A, Schulz AK, Hejnol A, Meinhardt $H$ et al. Global cell sorting in the $C$. elegans embryo defines a new mechanism for pattern formation. Dev Biol 2006; 294: 418-431. 